\title{
Magnitude, Seasonal-variation, Serological and Hematological Profile of Dengue in a Tertiary Teaching Hospital, Karwar, India
}

\author{
F. Sneha Kukanur ${ }^{1}$ (D) G. Naveen ${ }^{1}$ (D) N. Ashwin Chitrabanu²* (D), \\ B.M. Prashant ${ }^{3}$, R. Meghana ${ }^{1}$ and V.N. Venkatesh ${ }^{1}$ \\ ${ }^{1}$ Department of Microbiology, Karwar Institute of Medical Sciences, Karwar, Uttara Kannada - 581 301, \\ Karnataka India. \\ ${ }^{2}$ Karwar Institute of Medical Sciences, Karwar, Uttara Kannada - 581 301, Karnataka, India. \\ ${ }^{3}$ Department of Pathology, Karwar Institute of Medical Sciences, Karwar, \\ Uttara Kannada - 581 301, Karnataka, India.
}

\begin{abstract}
Dengue viral infection is the most widely spread arbo-viral disease in Indian subcontinent. High index of clinical suspicion especially during its peak season can be rewarding in diagnosing as well as early case management of anticipated DHF and DSS cases. To estimate the magnitude, seasonal-variation, serological as well as hematological aspects of dengue cases. This was a prospective observational study held in Microbiology and Hematology laboratories of our hospital for duration of one year from July-2019 to June-2020. All the suspected dengue cases were subjected to NS1-antigen, IgM and IgG antibody detection. The samples were also tested for platelet count, total count, haematocrit as well as hemoglobin estimation. All 1,550 dengue suspected cases were subjected to serological testing, among which $157(10.1 \%)$ were positive. The most affected populations were the adult male. As the study was conducted for one year, we could observe the seasonal trend which peaked during post-monsoon. Out of 157 cases, $81.5 \%, 0.6 \%$ and $17.8 \%$ were determined as primary, secondary and old dengue cases respectively. There was a significant association between NS1 antigen and fever of $</=5$ days duration with ' $p$ ' value $<0.00001$. Thrombocytopenia, leucopenia and increased haematocrit were witnessed in $15.9 \%, 28.6 \%$ and $35 \%$ respectively. Our study shows that we had a high magnitude of primary cases that are prone to secondary dengue infection which might have a catastrophic effect giving rise to DHF, DSS or SD.
\end{abstract}

Keywords: Dengue, Early-primary case, Late-primary case, Seasonal-variation, Thrombocytopenia, Haematocrit 15(4):1892-1897. doi: 10.22207/JPAM.15.4.10

(C) The Author(s) 2021. Open Access. This article is distributed under the terms of the Creative Commons Attribution 4.0 International License which permits unrestricted use, sharing, distribution, and reproduction in any medium, provided you give appropriate credit to the original author(s) and the source, provide a link to the Creative Commons license, and indicate if changes were made. 


\section{INTRODUCTION}

Dengue alone contributes to a major chunk of arthropod borne arboviruses in India. It spreads through the bite of infected female Aedes aegypti mosquito. ${ }^{1}$ Population explosion, poor waste management, lack of proper drainage systems, migration, globalization, rapid urbanization, deforestation are the few reasons that dengue has made its place as one of the fastest emerging viral infections. ${ }^{2}$

In India dengue is one of the top endemic viral diseases. ${ }^{3}$ Its incidence exhibits seasonal variation. The incidence starts to increase in June, reaches its peak in post monsoon season of September, October and slowly falls thereafter., ${ }^{1,-6}$

Dengue has a wide variety of clinical manifestations because of which the clinicians should have high suspicion especially during the above season. ${ }^{2}$ According to WHO guidelines it is classified into dengue without warning signs, dengue with warning signs and severe dengue which includes dengue hemorrhagic fever and dengue shock syndrome. ${ }^{7}$

As there are no effective vaccines or specific antiviral drugs available, the successful strategy to tame dengue lies in timely and rapid detection of cases, case management and vector control. ${ }^{3,4,8}$ Virus isolation, immunological testing, and the molecular-based polymerase chain reaction have all been used in the past to diagnose dengue fever (PCR). These procedures, on the other hand, are expensive, take a long time to complete, and need specialized equipment and expertise. ${ }^{16}$ Rapid diagnostic tests (RDTs) to detect dengue NS1 antigen, IgM, and IgG antibodies have been developed commercially in recent years for faster detection as they provide results within 30 minutes. ${ }^{17,18}$ In consideration of the above and the scarce data available about the age and sex distribution, burden, seasonal variation as well as the serological and hematological profile of dengue from this locality, this study was conducted to answer the above research questions.

\section{MATERIALS AND METHODS}

This prospective observational study was carried out in the Microbiology as well as hematology laboratories from July 2019 to June 2020. The study was approved by the Institutional
Ethical Committee of Karwar Institute of Medical Sciences, Karwar.

The study population included all clinically suspected Dengue cases. Following collection of demographics, other details such as the day of fever and symptoms were collected, as well as any comorbidities.

A fresh blood samples were collected in EDTA as well as in plain vacutainers and were immediately sent to hematology and serology sections respectively. In hematology section the blood was subjected to platelet count, WBC count, hematocrit and hemoglobin estimation. All abnormal platelet count and WBC count were cross checked manually. Platelet count of less than $1,00,000$ cells/cu.mm was considered as thrombocytopenia and increased hematocrit was considered if the value was $\geq 45 \%{ }^{6}$

The serum samples received in serology section were subjected to NS1 antigen detection, IgM and IgG antibody detection by Dengucheck combo kit (immunochromatography method) as per manufacturer's instruction 15, 18. Interpretation of serology tests was done as dictated in Table 1. Any sample which was NS1 positive was further tested by ELISA method. ${ }^{4-6}$

Patient details and other data were entered and analyzed in Microsoft Excel sheet ensuring confidentiality. Collected data were analyzed and processed using SPSS v 16 . Chi square test was used to find a significant ${ }^{2}$ association between variables. ' $t$ ' test was used to observe a significant difference between the mean values. It was considered to be significant if ' $p$ ' value was less than 0.05 .

Table 1. Serological tests and their possible interpretations ${ }^{4}$

\begin{tabular}{ll}
\hline Test results & Interpretations \\
\hline $\begin{array}{ll}\text { NS1, IgM and IgG all } \\
\text { non reactive }\end{array}$ & Not dengue case \\
NS1reactive & Early primary case \\
IgM reactive & Late primary case \\
NS1+ IgM reactive & \\
NS1+ IgG reactive & Early secondary case \\
IgM+lgG & Late secondary case \\
NS1+ IgM+ IgG & Old case \\
IgG & \multicolumn{1}{c}{ www.microbiologyjournal.org }
\end{tabular}




\section{RESULTS}

Out of 1,550 clinically suspected dengue cases, 157 (10.1\%) were positive for one or the other serological tests specific for dengue.Among them 105 were males and 52 were females as shown in Table 2. Male to female ratio was found to be $2: 1$.

When looked at the age distribution among positive cases $60(38.2 \%)$ of them belonged

Table 2. Gender distribution among the positive dengue cases

\begin{tabular}{lc}
\hline Gender & Total (\%) \\
\hline Male & $105(67)$ \\
Female & $52(33)$ \\
Total & $157(100)$ \\
\hline
\end{tabular}

Table 3. Age distribution among dengue positive cases

\begin{tabular}{lc}
\hline $\begin{array}{l}\text { Age distribution } \\
\text { (in years) }\end{array}$ & $\begin{array}{c}\text { Total cases } \\
\text { (\%) }\end{array}$ \\
\hline 1 to 10 & $1(0.6)$ \\
11 to 20 & $29(18.5)$ \\
21 to 30 & $60(38.2)$ \\
31 to 40 & $25(15.9)$ \\
41 to 50 & $20(12.7)$ \\
51 to 60 & $12(7.6)$ \\
61 to 70 & $7(4.5)$ \\
71 to 80 & $1(0.6)$ \\
81 to 90 & $2(1.3)$ \\
Total & $157(100)$ \\
\hline
\end{tabular}

to the age group of 21 to 30 years as represented in Table 3. The mean age was 32.93 years.

The cases slowly started rising from June, reached peak in September and October and began decreasing from then as depicted in Table 4 and Fig. 1.

Among 157 cases, 128 (81.5\%) of them were Primary cases either early or late, only one $(0.6 \%)$ was secondary case and the rest $(17.8 \%)$ were old cases of dengue as shown in Table 5.

Table 6 represents the correlation between fever and positive serological tests. NS1 antigen with or without IgM was positive in 68 (43.3\%) patients with fever less than 5 days. IgM

Table 4. Seasonal variation of dengue cases

\begin{tabular}{lccc}
\hline Month & $\begin{array}{c}\text { Total } \\
\text { tested }\end{array}$ & Positive & $\begin{array}{c}\text { Percentage } \\
(\%)\end{array}$ \\
\hline July & 176 & 15 & 9.55 \\
August & 252 & 25 & 15.92 \\
September & 385 & 38 & 24.20 \\
October & 236 & 36 & 22.93 \\
November & 154 & 15 & 9.55 \\
December & 106 & 16 & 10.19 \\
January & 81 & 5 & 3.18 \\
February & 77 & 4 & 2.55 \\
March & 43 & 1 & 0.64 \\
April & 17 & 1 & 0.64 \\
May & 10 & 0 & 0.00 \\
June & 13 & 1 & 0.64 \\
Total & 1550 & 157 & 100 \\
\hline
\end{tabular}

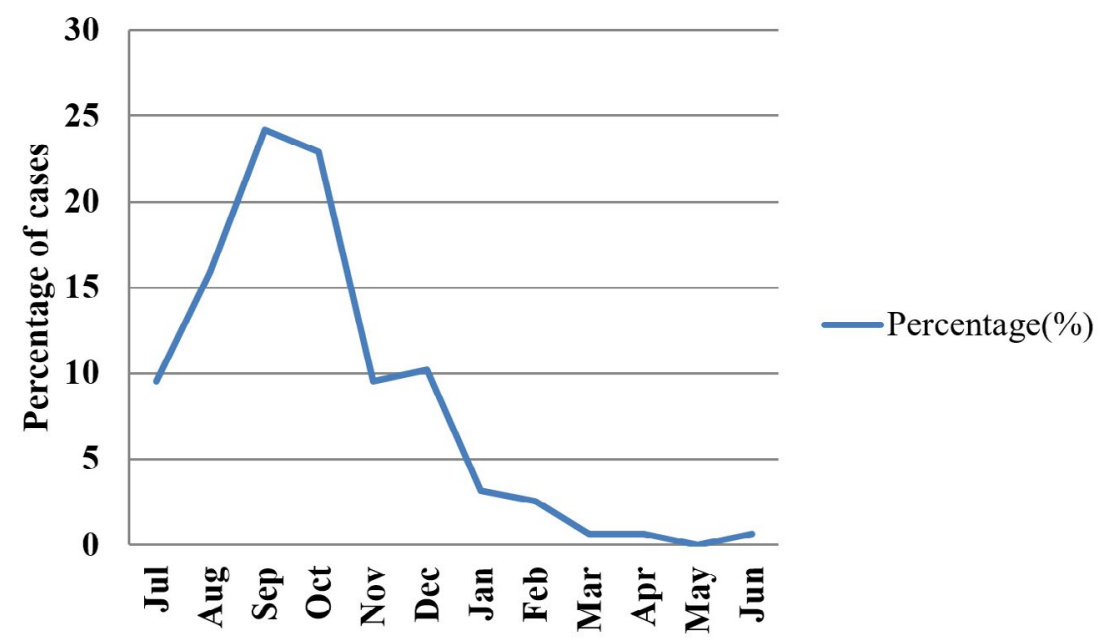

Fig. 1. Seasonal variation of Dengue cases. 
Table 5. Serological tests among positive dengue patients

\begin{tabular}{lcc}
\hline $\begin{array}{l}\text { Serological } \\
\text { tests }\end{array}$ & $\begin{array}{c}\text { Total positive } \\
(\%)\end{array}$ & Interpretation \\
\hline NS1 & $67(42.7)$ & Early Primary case \\
IgM & $46(29.3)$ & Late Primary case \\
NS1 + IgM & $15(9.5)$ & Late Primary case \\
IgM + IgG & $1(0.6)$ & Late Secondary case \\
IgG & $28(17.8)$ & Old dengue case \\
Total & $157(100)$ & Total dengue cases \\
\hline
\end{tabular}

Table 6. Correlation between the number of days of fever and serological tests

\begin{tabular}{lccc}
\hline \multirow{2}{*}{$\begin{array}{l}\text { Serological } \\
\text { tests }\end{array}$} & \multicolumn{2}{c}{ Number of positives (fever) } & \multirow{2}{*}{ Total } \\
\cline { 2 - 3 } & $</=5$ days & $>5$ days & \\
\hline NS1 & 67 & 0 & 67 \\
IgM & 2 & 44 & 46 \\
NS1 + IgM & 1 & 14 & 15 \\
IgM + IgG & 0 & 1 & 1 \\
IgG & 18 & 10 & 28 \\
\hline
\end{tabular}

with or without NS1 and IgG was positive in 59 $(37.5 \%)$ patients with fever more than 5 days. There was a significant association seen between patients with less than 5 days of fever and positive NS1 by both ELISA and serology ( $p<0.00001)$. A significant correlation was also derived between patients with more than 5 days of fever and positive IgM serology $(p<0.00001)$. While there were no significant association between IgG and fever days.

Table 7 depicts the hematological parameters in serologically positive cases with respect to days of fever. Thrombocytopenia, leucopenia and haemoconcentration (increased hematocrit value) were found only in $15.9 \%, 28.6 \%$ and $22.3 \%$ of the positive cases respectively.

\section{DISCUSSION}

Karwar being a coastal town relatively has higher humidity and receives lot of rain causing water stagnation at most places due to which mosquitoes can multiply, become infected, and spread disease very easily. Accordingly, every fever of unknown origin or undifferentiated fever case should be suspected as dengue unless proved otherwise especially during its peak season. ${ }^{2}$
Table 7.- Hematological parameters in Dengue positive patients

\begin{tabular}{|c|c|c|c|}
\hline $\begin{array}{l}\text { Haematological } \\
\text { parameter }\end{array}$ & $\begin{array}{l}\text { Frequency } \\
(\%)\end{array}$ & $\begin{array}{l}</=5 \\
\text { days }\end{array}$ & $\begin{array}{l}>5 \\
\text { days }\end{array}$ \\
\hline \multicolumn{4}{|c|}{ Platelet count (cells / cu.mm) } \\
\hline$<1,00,000$ & 25 (15.9) & 9 & 16 \\
\hline$>/=1,00,000$ & $132(84.1)$ & 59 & 53 \\
\hline \multicolumn{4}{|c|}{ WBC count (cells / cu.mm) } \\
\hline$</=4,000$ & $45(28.6)$ & 35 & 10 \\
\hline$>4,000$ & $112(71.3)$ & 53 & 59 \\
\hline \multicolumn{4}{|l|}{ Haematocrit value } \\
\hline$<45$ & $122(77.7)$ & 67 & 55 \\
\hline$>/=45$ & $35(22.3)$ & 21 & 14 \\
\hline \multicolumn{4}{|c|}{ Haemoglobin values (gm\%) } \\
\hline$<10$ & $7(4.5)$ & 1 & 6 \\
\hline$>/=10$ & $150(95.5)$ & 87 & 63 \\
\hline
\end{tabular}

In the present study 1,550 patients were suspected of dengue infection, however only 157 (10.1\%) was diagnosed of dengue infection. In a study by Goswami et al., ${ }^{5}$ the percentage of dengue infection (20\%) is a little higher compared to this study. This may be because increased awareness among our treating physicians and general public about the disease which gives rise to increased test requests. ${ }^{2,6}$

A male preponderance of (67\%) with the gender ratio being 2:1 was noted which was similar to a study done by T Damodar et al. ${ }^{2,19}$ When it comes to age distribution, most of them infected belonged to the age between 11 and 50 . Other studies also mention that adults were more affected than children and elderly population. ${ }^{3,5}$ This may be because the adult population is the most active. The productive time of the life where they tend to go out for work, market and many other purposes during day time exposes them to infection.10 Stoddard S T et al. state that, transmission rate is as high as 3.75 times if moved out of home as compared to 1.3 times when stayed at home. ${ }^{11}$

Dengue cases are found all throughout the year, but reach to the peak during post monsoon. This seasonal variation is observed in the present study as well as other studies. ${ }^{1,4-6}$. This trend can be explained because of high relative humidity and warm environment which helps the growth of vector. ${ }^{12}$

The varied clinical presentation of dengue has made clinical diagnosis difficult. Hence, the 
responsibility or burden of diagnosing dengue cases depends on standard and well equipped laboratory. ${ }^{9}$ There are mainly three methods for laboratory diagnoses of dengue infection, virus isolation on cell culture which is the gold standard, detection of viral RNA and serology which includes rapid tests as well as ELISA. ${ }^{9}$ Other supportive laboratory tests are platelet count, total WBC count, hematocrit and liver function tests to name a few. ${ }^{13}$

Virus isolation is expensive and takes nearly 10 days. Viral RNA detection needs well equipped laboratory and also dedicated staff, above so is also a costly affair. This narrows down our option for diagnosis to serological immunochromatographic rapid test or ELISA. ${ }^{9}$

Serological tests involve the detection of NS1 antigen, IgM and IgG antibodies specific for dengue. Test for NS1 antigen does not show any cross reaction with Japanese encephalitis virus, yellow fever virus or any other related viruses of Flavividridae family. This makes NS1 detection a reliable and key test to diagnose early dengue infection. . $^{3,14}$

Levels of sero-markers with respect to duration of dengue are as follows, NS1 antigen is detectable up to 9 days from the onset of infection. IgM antibodies start rising by 3-5 days and peak in 2 weeks and decrease to undetectable levels by another 3 months. By the end of the first week of infection the last sero-marker IgG antibodies start rising and are detectable for life. ${ }^{4}$

In the present study, $81.5 \%$ of cases were primary dengue cases, which include early as well as late infection, in contrast to $26 \%$ in a study by Bhattacharya et al. Only one case $(0.6 \%)$ in the present study was a secondary dengue infection while this proportion was $5.9 \%$ in study by Bhattacharya et al. A total of $17.8 \%$ of cases in the present study were IgG positive (old dengue cases) while Bhattacharya et al. had $34.2 \%{ }^{4}$ Table 8 shows that the present study has higher numbers of primary cases who are at high risk of acquiring secondary dengue infection who are prone to manifest as Dengue hemorrhagic fever (DHF), Dengue shock syndrome (DSS), Severe Dengue (SD) or atypical dengue infection.

A highly significant association between positive NS1 antigen and presentation of fever with less than 5 days duration is seen in the present study as depicted in Table 6 which is comparable to results of the study from Mangalore. ${ }^{2}$

The present study had thrombocytopenia in $16 \%$ of cases when compared to the studies done by T Damodar et al. and Ritu Karoli et al. they claimed to have $72 \%$ and $89 \%$ respectively. ${ }^{2,13}$ This study had few cases of leucopenia (28.6\%) when compared to $86 \%$ of the same in a study by Ritu Karoli et al. This difference might be because the present study had very less numbers of secondary cases. Thrombocytopenia, leucopenia and hemoconcentration are the phenomena that more frequently occur in secondary cases especially in DHF, DSS and SD. ${ }^{13}$

A few limitations of the present study such as the study were conducted in a one center, RT-PCR and Virus isolation in cell culture adopted could have increased the specificity of our study. ${ }^{6,9}$ Adept clinical details of the patients could help us to correlate laboratory findings with clinical manifestations better. ${ }^{2}$ Serotyping could help determine the epidemiological serotype in this locality and help the clinicians anticipate the complications and plan better patient management. $^{2}$

\section{CONCLUSION}

The study shows relatively higher burden of primary dengue cases, which imply that dengue is an emerging disease in this area. All the primary cases are at potential risk of getting infected by other serotype of dengue.

\section{ACKNOWLEDGMENTS}

The authors are thankful to the Department of Microbiology at Karwar Institute of Medical Sciences, Karwar, Karnataka, India.

\section{CONFLICT OF INTEREST}

conflict of interest.

The authors declare that there is no

\section{AUTHORS' CONTRIBUTION}

All authors listed have made a substantial, direct and intellectual contribution to the work, and approved it for publication

\section{FUNDING}

None 


\section{DATA AVAILABILITY}

All datasets generated or analyzed during this study are included in the manuscript.

\section{ETHICS STATEMENT}

The study was conducted after obtaining institutional ethical committee clearance from Karwar Institute of Medical Sciences, Karwar, Karanataka, India.

\section{REFERENCES}

1. Ganeshkumar P, Murhekar MV, PoornimaV, et al. Dengue infection in India: A systematic review and meta-analysis. PLoSNegl Trop Dis. 2018;12(7):0006618. doi: 10.1371/journal.pntd.0006618

2. Damodar T, Dias M, Mani R, et al. Clinical and laboratory profile of dengue viralinfections in and around Mangalore, India. Indian J Med Microbiol. 2017;35(2):256-261. doi:10.4103/ijmm.IJMM_15_423

3. Jahnavi K, Sreenivasulu T. Study of incidence, manifestations and complications of dengue fever. Int J Adv Med. 2018;5(1):137-140. doi: 10.18203/23493933.ijam201180072

4. Bhattacharya N, Mukherjee $\mathrm{H}$, Naskar R, et al. Serological diagnosis of dengue in laboratory practice in Kolkata. Indian J Med Microbiol. 2014;32(3):277280. doi: 10.4103/0255-0857.136563

5. Goswami L, Runumi C, Rasul ES. Seroprevalence of dengue infection in a tertiary care Hospital in Assam. Int J Med Dent Sci. 2018;7(1):1582-1585. doi: 10.18311/ijmds/2018/18905

6. Tewari VV, Tewari K, Mehta R. Clinical and hematological profile of patients with dengue fever at a tertiary care hospital - an observational study. Mediterr J Hematol Infect Dis. 2018;10:2018021. doi: 10.4084/ mjhid.2018.021

7. Guidelines For Diagnosis. New edition. Who.int. Accessed September 22, 2021. http://apps.who.int/ iris/bitstream/handle/10665/44188/9789241547871_ eng.pdf;sequence $=1$

8. Ministry of Health, Family Welfare-Government of India. Dengue. Gov.in. Accessed September 22, 2021. https://nvbdcp.gov.in/index1. php?lang=1\&level $=1 \&$ sublinkid $=5776 \&$ lid $=3690$
9. Anand AM, Sistla S, Dhodapkar R, Hamide A, Biswal $\mathrm{N}$, Srinivasan B. Evaluation of NS1 Antigen Detection for Early Diagnosis of Dengue in a Tertiary Hospital in Southern India. J Clin Diagn Res. 2016;10(4):DC01-DC4. doi: 10.7860/JCDR/2016/15758.7562

10. Halstead S. Recent advances in understanding dengue. F1000Res. 2019;8:F1000 Faculty Rev-1279. doi: 10.12688/f1000research.19197.1

11. Stoddard ST, Forshey BM, Morrison AC, et al. House-to-house human movement drives dengue virus transmission. Proc Natl Acad Sci U S A. 2013;110(3):994-999. doi: 10.1073/pnas.1213349110

12. Roy MP, Gupta R, Chopra N, Meena SK, Aggarwal KC. Seasonal variation and dengue burden in paediatric patients in New Delhi. J Trop Pediatr. 2018;64(4):336341. doi: 10.1093/tropej/fmx077

13. Karoli R, Fatima, Siddiqi Z, Kazmi KI, Sultania AR. Clinical profile of dengue infection at a teaching hospital in North India. J Infect Dev Ctries. 2012;6(7):551-554. doi: $10.3855 /$ jidc. 2010

14. Lapphra K, Sangcharaswichai A, Chokephaibulkit K, et al. Evaluation of an NS1 antigen detection for diagnosis of acute dengue infection in patients with acute febrile illness. Diagn Microbiol Infect Dis. 2008;60(4):387-391. doi: 10.1016/j.diagmicrobio.2007.11.010

15. Shah PD, Mehta TK. Evaluation of concurrent malaria and dengue infections among febrile patients. Indian J Med Microbiol. 2017;35(3):402-405. doi: 10.4103/ ijmm.IJMM_15_455

16. Wiwanitkit $\bar{V}$. Dengue fever: diagnosis and treatment. Expert Rev Anti Infect Ther. 2010;8(7):841-845. doi: 10.1586/eri.10.53

17. Blacksell SD. Commercial dengue rapid diagnostic tests for point-of-care application: recent evaluations and future needs? J Biomed Biotechnol. 2012;2012:151967. doi: 10.1155/2012/151967

18. Yow K-S, Aik J, Tan EY-M, Ng L-C, Lai YL. Rapid diagnostic tests for the detection of recent dengue infections: An evaluation of six kits on clinical specimens. PLOS ONE. 2021;16(4):e0249602. doi: 10.1371/journal. pone. 0249602

19. Prabowo MH, Chatchen S, Rijiravanich P, et al. Clinical evaluation of a developed paper-based Dengue NS1 rapid diagnostic test for febrile illness patients: Int J Infect Dis. 2021;107:271-277. doi: 10.1016/j. ijid.2021.05.007 